\title{
Institucionalizar la escritura del pasado. La Academia Paraguaya de la Historia $(1937-1965) *$
}

\author{
Institutionalize Writing Past. The Paraguayan Academy \\ of History (1937-1965)
}

Liliana M. Brezzo

CONICET, IDEHESI

Pontificia Universidad Católica Argentina

Este artículo analiza el proceso de fundación de la Academia Paraguaya de la Historia (antes Instituto Paraguayo de Investigaciones Históricas) y su vida institucional como vía para dar cuenta del desarrollo de la escritura de la historia en Paraguay en el siglo XX. Pretende distinguir los condicionantes en la práctica de la historia y examinar el rol de la Academia en el proceso de institucionalización de la disciplina.

PAlabras Clave: Paraguay; Institucionalización de la Historia; Discursos históricos.

This article analyzes the process of foundation and institutional life of the Paraguayan Academy of History (formerly Paraguayan Institute of Historical Research) as a way to account for the development of writing in Paraguay's History in the twentieth century. Attempt to distinguish the conditioning factors on the practice of History and examine the role of the Academy in the process of institutionalization of the discipline.

KEYwORDS: Paraguay; Institutionalization of History; Historical discourses.

* Deseo dedicar este estudio a Olinda Massare de Kostianovsky, presidenta de la Academia Paraguaya de la Historia, quien al momento de iniciarse esta investigación, en el año 2011, dispuso toda clase de facilidades para la consulta del archivo de la institución. Su inesperado fallecimiento, el 4 de diciembre de 2012, le impidió seguir los progresos de este trabajo. 
La historia de la historiografía paraguaya contemporánea es un campo apenas abonado hasta hoy a pesar del interés que, parece indudable, ofrece. ${ }^{1}$ Este trabajo propone centrar la atención en el proceso de fundación de la Academia Paraguaya de la Historia (antes Instituto Paraguayo de Investigaciones Históricas) y en su vida institucional como vía para dar cuenta de los itinerarios que la escritura de la historia ha descrito en Paraguay durante el siglo XX. Durante sus casi ochenta años de existencia, desde su creación en 1937, la corporación ha reunido a más de un centenar de miembros de número, entre los cuales figuran muchos — ciertamente que no todos- de los más notables exponentes de la disciplina histórica del país. ${ }^{2}$ ¿En qué circunstancias debe comprenderse la creación del Instituto Paraguayo de Investigaciones Históricas? ¿Quiénes fueron sus fundadores? ¿Cuáles sus intereses de estudio? ¿Qué peso tuvieron en la producción histórica de la época?

Un primer asunto en el que parece conveniente detenerse es el contexto en el que se establecieron las primeras asociaciones letradas que pusieron de manifiesto una preocupación por el estudio de la historia paraguaya.

\section{Aislacionismo, guerra y letrados}

La trayectoria de la historiografía paraguaya está delimitada, en parte, por su poliédrica realidad aislacionista. En efecto, la situación geográfica de un país en la periferia extrema de la frontera interior sudamericana —una «isla rodeada de tierra», como lo definió Augusto Roa Bastoshace presuponer una amplia separación del resto del mundo occidental. Luego, las circunstancias políticas en las que se encontró el Paraguay después del año 1811, en el que se produjo la revolución de la independencia, hicieron difíciles las prácticas normales del comercio, lo cual inevitable-

1 Para la historia de la historiografía paraguaya no disponemos aún de estudios que tracen un panorama general de su evolución. Como instrumento de aproximación sigue siendo valiosa la reseña de Williams, 1973. Una perspectiva actualizada, aunque parcial, en Brezzo, 2010a. El encomiable y exhaustivo trabajo de Cardozo, en 1959, se limita a la producción historiográfica correspondiente al período colonial.

2 El cada vez menos exiguo interés por el rol que las Academias han tenido en la profesionalización e institucionalización de la historia se ha puesto de manifiesto en estudios recientes que proyectan un haz de luz importante y que han servido para el planteo teórico de este trabajo. Entre esos destaco los de Nava Rodríguez, 1987; Vázquez, 2001; Hampe Martínez, 1998; Betancourt Mendieta y Ramírez Bacca, 2009; Betancourt Mendieta, 2007; Devoto y Pagano, 2009; Micheletti, 2013. 
mente dificultó el paso de ideas y de bienes. El ascenso al poder de José Gaspar Rodríguez de Francia (1814-1840), quien colocó un «cordón político» en torno al país para «protegerlo del caos del sur» y practicó una política internacional de enclaustramiento, contribuyó a su separación del resto de los países del Río de la Plata. ${ }^{3}$ En razón de ese aislamiento y del aislacionismo, el Paraguay era a mediados del siglo XIX prácticamente un país desconocido, que incitaba la curiosidad de viajeros europeos y americanos y suscitaba una figura alegórica que recuerda lo que hoy se conoce como «formaciones asiáticas»: el aislamiento del mundo exterior; el control de una parte de su producción y explotación de recursos económicos por un lado, y del comercio exterior por el otro, ejercido por el Estado; la existencia de un poder centralizado, autoritario y vitalicio en la práctica; y la veneración cuasi religiosa de ese supremo gobierno por una población masivamente campesina, proporcionaban elementos a sumarias definiciones que hacían del Paraguay «la China, el Japón de América». Esta realidad condicionó que, si bien se divulgaron estudios sobre la historia y la geografía del país, casi sin excepción se trataron de relatos de viajeros, diplomáticos o escritores extranjeros; de hecho, los registros disponibles indican que desde el texto de Ruy Díaz de Guzmán, ${ }^{4}$ escrito a comienzos del siglo XVII, hasta finales del XIX la historia paraguaya fue redactada fundamentalmente por autores foráneos. ${ }^{5}$ Sin desconocer ese fuerte condicionante, investigaciones recientes han demostrado la unilateralidad de la perspectiva aislacionista para caracterizar ese itinerario historiográfico. Entre estas destacan las dedicadas a la identificación y edición de escritos producidos por letrados paraguayos durante la primera mitad del siglo XIX, como los de José Falcón, Juan José Brizuela y Luciano Recalde. Inéditos hasta el presente, sus textos aparecen como expresión de las «luces atenuadas» que el país mostraba en esa época mostrando que, aunque insertos en un mundo cultural diminuto, en el Paraguay decimonónico existían quienes encarnaban la «resistencia» a la realidad aislacionista, exteriorizando un interés en el mundo más amplio, formulándose preguntas sobre sí mismos y sobre el pasado y presente de su sociedad. ${ }^{6}$

3 Brezzo, 2010b

4 Ruy Díaz de Guzmán (1560-1612), nació en Asunción, nieto de Domingo de Irala y de madre guaraní. En 1612 fechó su Historia del Descubrimiento, Conquista y Población del Río de la Plata. Para un itinerario del códice hasta su primera edición en 1836, véase Cardozo, 1959, 85-90.

5 Vidaurreta, 1972.

6 Whigham y Scavone Yegros, 2006; Scavone Yegros, 2010b. 
Consta asimismo que los gobiernos de Carlos Antonio López (18421862) y de Francisco Solano López (1862-1870) se preocuparon por impulsar el desarrollo cultural del país. En 1856 quedó establecida en Asunción el Aula de Filosofía, que reunió a medio centenar de estudiantes bajo la dirección del profesor español Ildefonso Antonio Bermejo, quien había arribado al país contratado por el Gobierno para organizar una Escuela Normal. En el Aula, que pretendía constituirse en base de un centro universitario del que Paraguay carecía desde la época colonial, surgió el grupo de redactores que se responsabilizaría de la edición de La Aurora. Enciclopedia mensual y popular de ciencias, artes y literatura, primera revista cultural paraguaya, cuyas entregas comenzaron a aparecer en 1860. Al mismo tiempo, entre los años 1850 y 1860 el Congreso nacional autorizó al poder ejecutivo a enviar jóvenes con destino a Europa para proseguir estudios universitarios; unos 50 becarios paraguayos se instalaron en Inglaterra y en Francia para estudiar Derecho, Química y Farmacia. ${ }^{7}$ Mas todas estas iniciativas se vieron drásticamente interrumpidas por el inicio de la guerra contra la Triple Alianza (Argentina, Uruguay, Brasil, 1864-1870) y las consecuencias de la derrota determinaron por completo su evolución sociocultural: la población quedó reducida a un $30 \%$ (conformada fundamentalmente por niños, ancianos y mujeres) de los 400.000 habitantes con que contaba al comienzo de la contienda, el sistema educativo completamente desarticulado, los archivos estatales destruidos y las bibliotecas públicas y privadas diezmadas. ${ }^{8}$

Sobre el rescoldo todavía doloroso de la guerra, en medio de la extrema pobreza y de los esfuerzos para reconstruir el país se fue conformando una elite letrada a la que se conocería como la Generación del 900 o Novecentistas. Nacidos la década siguiente al conflicto, formados en su mayoría en el Colegio Nacional de Asunción (1877) y más tarde en la Facultad de Derecho de la Universidad Nacional (1889), entre sus principales exponentes estaban Blas Garay (1873-1899), Juan O'Leary (1879-1969), Manuel Domínguez (1868-1935), Fulgencio Moreno (1872-1933), Arsenio López Decoud (1867-1945), Ignacio Pane (1879-1920), Eligio Ayala (18791930), Manuel Gondra (1871-1927), Gualberto Cardús Huerta (1878-1949), Alejandro Guanes ( 1872-1925) y Teodosio González (1871-1932). ${ }^{9}$

7 Durán Estragó, 2006, 7-12; Schmitt, 1990, 88-91.

8 Brezzo, 2010a.

9 Una aproximación en torno al proceso que posibilitó la emergencia del grupo denominado Generación del 900 como así también a la fisonomía colectiva puede leerse en Gómez Lez y Zarza, 2013; Amaral, 2006; Centurión, 1961. 
Gracias a su iniciativa quedó constituido en 1895 el Instituto Paraguayo, la primera institución cultural de la posguerra. Sus impulsores se limitaron a enunciar como propósitos el fomento del estudio de la música y el desarrollo de la literatura, proporcionar la enseñanza de idiomas y estimular los ejercicios físicos por medio de la gimnasia y la esgrima. Sin embargo, las cuestiones sobre el pasado reciente del país pasaron a constituirse rápidamente en materia predominante de sus preocupaciones y actividades - principalmente conferencias y debates-, las que a partir de 1896 comenzaron a divulgarse en la Revista del Instituto Paraguayo. Definida como de carácter «esencialmente científico», poco tiempo después pasaría a subtitularse «Historia, Ciencias, Letras», haciendo referencia a sus principales contenidos y convirtiéndose en la principal publicación de erudición histórica de la época tanto por sus temáticas como por el esfuerzo que suponía su edición en el yermo contexto cultural..$^{10}$ Asimismo, en la primera década del siglo XX comenzaron a circular otras publicaciones con similares pretensiones, como la Revista de la Universidad Nacional y la Revista de Agronomía y de Ciencias Aplicadas, dirigida por el agrónomo suizo Moisés Bertoni, las que convivieron a su vez con las dirigidas a un público selecto pero más aligeradas, con grabados de paisajes y de personajes, anecdotarios y textos breves. El catálogo de la biblioteca de José Segundo Decoud, uno de los intelectuales y políticos más influyentes de ese tiempo, da noticias de algunas de ellas, de las cuales han quedado pocos o ningún ejemplar, como Ilustración Paraguaya, La Revista Cómica, La Rivista Italiana. Coloniale, comerciale, sociale y The Paraguay Review Paraguayische Nachrichten, Tribuna de los intereses del Paraguay, La Revista del Paraguay, Crónica y Anales del Gimnasio Paraguayo, todas publicadas entre la última década del siglo XIX y la segunda década del XX. ${ }^{11}$

En 1913 quedó establecido en Asunción el Gimnasio Paraguayo, dedicado a impulsar el estudio y la divulgación de la música, los idiomas y la práctica de la esgrima y de todo «elemento de cultura»; los Anales del Gimnasio Paraguayo tuvieron entre 1917 y 1924 similar programa editorial que la Revista del Instituto Paraguayo. Finalmente, en el año 1933, ambas

10 La Revista se editó entre 1896 y 1909, con un total de 64 números que incluyeron estudios históricos de importancia, como los de Blas Garay sobre la Primera Junta Gubernativa, la compilación de documentos jesuíticos inéditos y los textos del reconocido letrado Cecilio Báez sobre historia diplomática. Véase Brezzo, 2011.

11 Catálogo detallado, 1912. 
instituciones culturales se fusionaron en el Ateneo Paraguayo, prosiguiendo el propósito del «cultivo de las ciencias, de las letras y las bellas artes».

En ese contexto, los estudios históricos se vieron atravesados a su vez por la controversia de límites que el Paraguay mantenía con Bolivia por la zona del Chaco Boreal.

\section{Nuevos temas, una segunda guerra}

La disputa chaqueña entre Paraguay Bolivia fue agravándose entre las dos últimas décadas del siglo XIX y los primeros años del XX. En ocasión de las sucesivas negociaciones diplomáticas destinadas a finiquitarla, letrados adscriptos al novecentismo se dieron a la tarea de acopiar documentos históricos para probar la justicia de la demanda paraguaya. Debieron adentrarse, para la reconstrucción del itinerario jurídico, en temas de historia política y social remontándose hasta la época de la fundación y ocupación de la región guaraní en el siglo XVI. Entre ellos, Manuel Domínguez y Fulgencio R. Moreno destacarían por su profusa producción histórica, la que en parte sería el resultado de sus trabajos diplomáticos al frente de la legación paraguaya en Bolivia y de su actuación en la Comisión Nacional de Límites. La primera obra de Domínguez apareció en el año 1917 con el título Paraguay-Bolivia: cuestión de Límites. Y hasta el año de su fallecimiento, en 1935, publicaría más de una decena de monografías entre las que sobresalieron: El derecho de descubrir y conquistar el Paraguay o Río de la Plata (1918), El Chaco Boreal: informe que arruina las tesis bolivianas y expone los títulos del Paraguay sobre dicha zona (1918), El Chaco boreal fue, es y será nuestro (1925), Nuestros pactos con Bolivia (1928), Bolivia y sus mistificaciones (1932), El Chaco pertenecía al obispado de Paraguay (1933), Siete reyes y diez virreyes afirman los derechos del Paraguay sobre el Chaco (1933), Expediciones del Paraguay al Chaco (1934) у Bolivia atropelló el statu-quo y sus reconocimientos del laudo Hayes (1935). Por su parte, Fulgencio Moreno hizo visible su interés por el tema chaqueño a partir del año 1904 cuando publicó Diplomacia paraguayo-boliviana, interés que iría in crescendo en el contexto de la puja diplomática de los años veinte en cuyo transcurso publicó La extensión territorial del Paraguay al occidente de su río: breve exposición de los títulos (1925). Y en 1933, poco antes de su fallecimiento, editó Paraguay-Bolivia: cuestión de límites. 
Al mismo tiempo, en Buenos Aires, Enrique de Gandía (1904-2000) comenzó a interesarse también en la controversia por el Chaco. Hijo de un español y de una italiana, este letrado había estudiado Derecho en la Universidad Complutense de Madrid y Sociología en la Universidad de la Sorbona de París, circunstancias que le permitieron conocer importantes bibliotecas y archivos de los dos países. Su producción histórica había logrado destacarse y obtener reconocimiento en la capital argentina. Como resultado de ello, en 1930 fue elegido miembro de número de la Junta de Historia y Numismática Americana, predecesora de la Academia Nacional de la Historia.

En ese contexto, De Gandía inició contactos epistolares con Manuel Domínguez, Fulgencio Moreno y con Juan E. O'Leary ${ }^{12}$ y viajó a Sevilla para estudiar en el Archivo de Indias documentación referida al litigio. Con el sustento de los textos de los autores paraguayos y de los documentos acopiados por su cuenta concluyó sobre la justicia de la tesis paraguaya: el Chaco había sido explorado y conquistado por el Paraguay, que desde los orígenes de la conquista había ejercido el dominio ininterrumpido y pacífico. Los argumentos los desenvolvió en tres estudios principales: Historia del Gran Chaco, editado en Madrid en el año 1929, Limites de las gobernaciones sudamericanas en el siglo XVI, en 1933, y Los derechos del Paraguay sobre el Chaco Boreal y las doctrinas del uti possidetis en el siglo XVI, publicado en Buenos Aires en 1935.

A comienzos de los años treinta, la disputa entre Paraguay y Bolivia se complicó y la opinión pública de los dos países reclamó soluciones de fuerza. La guerra del Chaco, entre 1932 y 1935, constituyó una nueva sangría para el Paraguay, que estaba aún rehabilitándose tras el desastre de la anterior conflagración. Poco después de la finalización de las acciones militares y de la firma del Tratado de Paz definitivo, a mediados de 1937, De Gandía viajó a Paraguay.

12 República de Paraguay, Biblioteca Nacional del Paraguay, Colección Juan E. O’Leary, Sección Correspondencia Pública y Privada (BNP-CJEO). De Gandía y O’Leary desenvolvieron una fluida correspondencia que se inició con motivo de los artículos que este último publicaría en El Liberal a comienzos de la guerra y que le merecieron al letrado argentino los siguientes juicios: «Con publicaciones como la que Ud. reproduce, los bolivianos no hacen más que confesar la injusticia de sus pretensiones, lo erróneo de sus fundamentos, la endeblez de sus razones. Ni por casualidad, como ellos dicen, se levanta en Bolivia alguien a defender la causa boliviana. Es lógico, ¿qué hombre de algún talento se va a poner a defender un pleito mil veces perdido, histórica y jurídicamente, que ni siquiera inventando absurdos podría ganarse?». Buenos Aires, 10 de noviembre de 1933. 


\section{La fundación del Instituto de Investigaciones Históricas}

Como lo han subrayado investigaciones recientes, el final del conflicto bélico con Bolivia derivó, en Paraguay, en una situación política interna de difícil consolidación. ${ }^{13} 46.000$ paraguayos dejaron el frente militar tras la victoria, luego de que se suscribiera el Protocolo de Paz con Bolivia el 12 de junio de 1935. Habían integrado una organización eficaz que consiguió expulsar al enemigo del territorio en disputa, gracias a los esfuerzos de toda la población. Resultaba natural que se sintieran artífices potenciales de la grandeza de la patria y que confiasen en que, después de la conflagración, había llegado la hora de las grandes transformaciones. Más comprometidos se consideraron los jefes y oficiales, consustanciados con las aspiraciones de la ciudadanía que habían comandado. Este espíritu colectivo chocó, sin embargo, con la dura realidad. Las finanzas del Estado habían tocado fondo y el gobierno de Eusebio Ayala (1932-1936) debió, antes que nada, reajustar el gasto público, desmantelando la estructura montada para la guerra. Se dispuso la desmovilización de oficiales y soldados, sin compensaciones y sin acompañar su reinserción a la vida civil. La forma en que se ejecutó esta medida dejó la sensación de que se estaba cometiendo una injusticia. A ello se sumaban las pugnas del gobernante partido Liberal de cara a las elecciones presidenciales para el período 19361940, la angustiosa situación económica del país y los problemas sociales no resueltos.

Los dos partidos políticos tradicionales del Paraguay, el Liberal y la Alianza Nacional Republicana o partido Colorado, fueron fundados en 1887. Este último había monopolizado el poder de 1887 a 1904 cuando fue derrocado por la fuerza por el partido Liberal, reteniendo el control hasta 1936. En efecto, a principios de ese año, el presidente Ayala dispuso la detención y expatriación del coronel Rafael Franco, junto a otros oficiales, a quienes acusó de conspirar en su contra. La decisión adoptada contra el prestigioso jefe durante la guerra aceleró los afanes conspirativos de los mandos medios y la oficialidad joven del Ejército. El 17 de febrero estalló en Asunción un movimiento revolucionario contra el gobierno de Ayala, que pasó a denominarse revolución febrerista o febrerismo. Esencialmente de carácter militar, los sublevados difundieron un Acta Plebiscitaria en la que anunciaron el cese del personal de los tres poderes del Estado y la

13 Scavone Yegros, 2010a; Rodríguez, 2010. 
restitución de la nación paraguaya al nivel de su historia en el Río de la Plata, al libre dominio de su suelo y a la grandeza de su porvenir. Y eligieron a Franco como presidente provisional.

Pronto se produjeron fricciones entre los grupos que apoyaban al gobierno, es decir, los militares y los ex combatientes. El poder ejecutivo quedó bajo el predominio de los hombres de la Liga Nacional Independiente, un reducido grupo de intelectuales nacionalistas liderados por el canciller del gobierno revolucionario, Juan Stefanich. Ese protagonismo de la Liga, los esfuerzos del gabinete ministerial para contener las injerencias militares, las intensas campañas interna e internacional de desprestigio promovidas por los sectores de oposición, especialmente del partido Liberal, contra la gestión del coronel Franco y las alternativas de las negociaciones diplomáticas en la Conferencia de Paz del Chaco generaron un nuevo levantamiento. El 10 de agosto de 1937 el comandante de las tropas del Chaco, teniente coronel Ramón Paredes, intimó al presidente Franco para que se deshiciese del canciller Stefanich. No tuvo éxito y esa fue la señal para el alzamiento militar. El 12 de agosto la Marina y la prefectura se plegaron al movimiento con lo que se evitó el derramamiento de sangre al considerarse estéril toda resistencia. El presidente Franco fue apresado y luego se exilió en Montevideo.

Derrocado el febrerismo, la solución política gestada por los militares buscó el apoyo del partido Liberal, accediendo a la presidencia el respetado jurista Félix Paiva, decano de la Facultad de Derecho de la Universidad Nacional de Asunción, el 16 de agosto de 1937. Los ministerios fueron confiados en su mayoría a profesores de esa unidad académica: Cecilio Báez estuvo a cargo del ministerio de Relaciones Exteriores, Justo Pastor Benítez y Enrique Bordenave pasaron por el de Hacienda, Justo P. Prieto y Luis A. Argaña encabezaron, sucesivamente, el de Instrucción Pública y Andrés Barbero dirigió el ministerio de Economía. Por este motivo, se conoció a este conjunto como el «gabinete universitario».

Fue en esas circunstancias en las que Enrique de Gandía llegó a Asunción. El clima revolucionario se mezclaba con las celebraciones por el cuarto centenario de la fundación de la ciudad —el 15 de agosto- y con las públicas demostraciones religiosas que acompañaban la realización del Primer Congreso Eucarístico Nacional. Su defensa intelectual de la posición paraguaya durante la guerra y los vínculos interpersonales establecidos con influyentes letrados paraguayos explican las muestras de simpatía que se le prodigaron. Representantes de instituciones educativas, de la 
prensa y de la Universidad Nacional de Asunción fueron a recibirlo al puerto asunceno. Los principales diarios — La Hora, La Capital, El Día- le dedicaron artículos con títulos elocuentes: «Lo que Enrique de Gandía ha hecho por el Paraguay» y «El Alberdi de la Guerra del Chaco»; la Universidad Nacional lo nombró doctor honoris causa y, según su propio testimonio, durante los quince días que permaneció en Paraguay pronunció dieciséis conferencias. ${ }^{14}$

Poco después de su llegada, De Gandía reunió a un grupo de «amigos historiadores» en el Hotel Colonial y les propuso la fundación de una Academia o de un Instituto dedicado a investigaciones históricas, inclinándose la mayoría de los asistentes por esta última denominación. De este modo, el 15 de agosto de 1937 se levantó un acta y se declaró constituido el Instituto Paraguayo de Investigaciones Históricas (IPIH). Se designó presidente honorario, por unanimidad, a De Gandía, y se conformó una estructura directiva jerárquica presidida por Adolfo Aponte (1874-1949) e integrada por: Vicepresidente 1. ${ }^{\circ}$ Ramón I. Cardozo (1876-1943), Vicepresidente 2. ${ }^{\circ}$ Hipólito Sánchez Quell (1907-1986), Secretarios R. Antonio Ramos (1907-1984), Eduardo Amarilla Fretes (1908-1990) y Andrés Barbero (1877-1951). Entre los firmantes del acta de fundación figuran también el ministro de Instrucción Pública, Luis A. Argaña, y los letrados Carlos R. Centurión, Ramón Lara Castro, Viriato Díaz Pérez, Arturo Brugada y Gabriel Ruiz. En el mismo acto se resolvió designar como miembros correspondientes en el exterior a Luis Alberto de Herrera, en Uruguay; Ricardo de Lafuente Machaín y Juan Esteban Guastavino, en Argentina; Walter A. de Acevedo, en Brasil y Carlos E. Grez Pérez y Manuel Caviezes Serrano, en Chile..$^{15}$

El Instituto proponía:

Fomentar los estudios históricos en general y especialmente los que se refieren al Paraguay y su divulgación; unir a los estudiosos nacionales que investigan el pasado histórico del país y establecer relaciones intelectuales entre los historiadores paraguayos y los extranjeros. ${ }^{16}$

De igual modo contemplaba organizar conferencias, certámenes, sesiones públicas, editar obras históricas antiguas y modernas y una revista o boletín que sería el órgano oficial del Instituto.

14 Massare de Kostianovsky, 2007.

15 Idem.

16 Ibidem, 352. 
El primer estatuto, compuesto por 22 artículos, fijaba en 40 la cantidad de miembros de número y en diez la de miembros correspondientes, ambos de carácter vitalicio. Para hacer efectiva su incorporación bastaba que quien fuese nombrado aceptase el cargo dentro de los cuatro meses de recibida la comunicación. Desde ese momento quedaba incorporado y se le expediría el diploma correspondiente.

Carentes de una sede, los integrantes del Instituto se reunirían en los meses siguientes a la fundación en la casa particular de Aponte. Según las actas de sesiones, los primeros domingos de cada mes, por la mañana, los asistentes intercambiaban impresiones sobre los estudios históricos que cada uno tenía en progreso. De este modo las primeras asambleas aparecen más bien como una especie de cenáculo, más identificadas con una tertulia que con una academia.

Entre las primeras determinaciones de la comisión directiva estuvo desenvolver un programa de extensión cultural consistente en la realización de conferencias a cargo de los miembros del Instituto, abiertas al público y con una periodicidad quincenal. Se obtuvo que el Ateneo Paraguayo facilitara sus instalaciones; el 6 de octubre quedaron inauguradas las actividades previstas con una conferencia de Efraím Cardozo sobre las «Primeras Monedas en el Río de la Plata» y el día 12 del mismo mes Viriato Díaz Pérez disertó sobre «Juan de Salazar y Espinoza: el capitán poeta».

Como toda institución no oficial, el IPIH tenía dificultades financieras. Obtuvo para su funcionamiento subsidios esporádicos por parte del Estado. Por ejemplo, los ministerios de Instrucción Pública y Justicia y el de Gobierno y Trabajo le otorgaron partidas entre los años 1940 y 1942 para el pago de muebles y gastos de oficina. Consta en el acta de sesión del 17 de julio de 1940 que con esa ayuda se mandó confeccionar una mesa, 12 sillas para el salón biblioteca, una mesa escritorio y una silla giratoria para la presidencia, «todos de estilo español, en madera petiribí». No obstante esas asistencias pecuniarias no fueron suficientes. Debido a esa condición, el presidente Aponte solicitó al poder Ejecutivo la personería jurídica del Instituto, la que le fue otorgada por el Decreto N. 14.400 de fecha 31 de agosto de 1942. Se lo ponderó como un medio para allanar de algún modo la obtención de un fomento permanente que le permitiera sostener una mínima estructura administrativa y «comenzar con la edición de un Boletín o una Revista».

Pues bien ¿quiénes eran los letrados paraguayos que firmaron el acta de constitución del Instituto? Una aproximación sociológica a los 12 hom- 
bres que figuran como «fundadores» permite delinear algunos rasgos de la fisonomía del grupo, delimitar sus espacios de actuación profesional y su práctica de la historia.

\begin{tabular}{|l|c|c|c|c|c|c|c|c|c|c|c|c|}
\hline Formación académica & 1 & 2 & 3 & 4 & 5 & 6 & 7 & 8 & 9 & 10 & 11 & 12 \\
\hline Derecho & $*$ & $*$ & & & $*$ & $*$ & & $*$ & $*$ & & $*$ & \\
\hline Filosofía/Letras & & & & & & & & & & $*$ & & \\
\hline Educación & & & $*$ & & & & & & & & & $*$ \\
\hline Medicina & & & & $*$ & & & & & & & & \\
\hline Estructura socio-profesional & & & & & & & & & & & & \\
\hline Enseñanza superior & $*$ & $*$ & & $*$ & $*$ & $*$ & & $*$ & $*$ & $*$ & & \\
\hline Enseñanza media & & $*$ & $*$ & & & & & & $*$ & $*$ & & $*$ \\
\hline Abogado & & $*$ & & & & $*$ & & & & & & \\
\hline Médico & & & & $*$ & & & & & & & & \\
\hline Publicista & $*$ & $*$ & $*$ & $*$ & $*$ & $*$ & & & & $*$ & $*$ & \\
\hline Actividad pública & & & & & & & & & & & & \\
\hline Política nacional/local & & $*$ & $*$ & $*$ & $*$ & $*$ & & & & $*$ & $*$ & \\
\hline $\begin{array}{l}\text { Servicios oficiales/ } \\
\text { administración nacional }\end{array}$ & $*$ & $*$ & $*$ & $*$ & $*$ & $*$ & & $*$ & $*$ & $*$ & & \\
\hline
\end{tabular}

1: Enrique de Gandía (1904-2000); 2: Adolfo Aponte (1887-1949); 3: Ramón Indalecio Cardozo (18761943); 4: Andrés Barbero (1877-1951); 5: Hipólito Sánchez Quell (1907-1986); 6: R. Antonio Ramos (1907-1984); 7: Eduardo Amarilla Fretes (1908-1990); 8: Ramón Lara Castro (1873-1958); 9: Carlos R. Centurión (1902-1969); 10: Viriato Díaz Pérez (1875-1958); 11: Arturo Brugada (1895-1939); 12: José Gabriel Ruiz (1894-1949).

En primer término hay que advertir que en los años treinta no existía aún en Paraguay enseñanza universitaria de la historia. En su mayor parte los fundadores del Instituto procedían de la Facultad de Derecho de la Universidad Nacional de Asunción (como son los casos de Adolfo Aponte, Hipólito Sánchez Quell, R. Antonio Ramos, Ramón Lara Castro, Carlos R. Centurión y Arturo Brugada); también en su mayoría ejercían la docencia media y/o universitaria (Ramón I. Cardozo enseñaba Historia en el Colegio 
Nacional de Asunción, J. Gabriel Ruiz dictaba Historia del Paraguay en el Colegio Nacional de Comercio, Hipólito Sánchez Quell estaba a cargo de la cátedra de Historia Diplomática en la Universidad Nacional de Asunción, Carlos R. Centurión enseñaba Historia en la Escuela Normal de profesores y luego Derecho Internacional Público en la Facultad de Derecho). Algunos tuvieron cargos en la administración nacional: R. Antonio Ramos fue director del Archivo Nacional en tanto Viriato Díaz Pérez fue director de la Biblioteca Nacional y jefe del archivo. Mayoritariamente ejercieron cargos dentro de la política nacional: ministro de Justicia, Culto e Instrucción Pública (Adolfo Aponte) y de Economía (Andrés Barbero); representante en las Conferencias de Paz con Bolivia (R. Antonio Ramos); jefe de legación diplomática (Lara Castro fue ministro plenipotenciario en Brasil en 1912 y luego entre 1918 y 1920) y diputado en el Congreso Nacional (Carlos R. Centurión). Y, sin excepciones, tenían una activa participación en la prensa: escribían en El Diario, El Liberal, Democracia y Patria.

La mayoría de estos hombres estaban afiliados al partido Liberal (Aponte, Barbero, Cardozo, Ramos, Lara Castro, Centurión, Chaves) y menos al partido Colorado (Sánchez Quell y Brugada).

\section{La primera etapa}

Las actas de sesiones y la correspondencia pública del IPIH permiten identificar una primera etapa comprendida entre los años 1937 y 1956, correspondiente a las presidencias de Adolfo Aponte (1937-1949) y de Ramón Lara Castro (1949-1956), signada por el desenvolvimiento de un plan de acción en dos direcciones. La primera consistió en el fomento de vínculos con corporaciones similares de la región. Para ello se intercambiaron notas con el Instituto Histórico y Geográfico de Río de Janeiro, la Junta de Historia y Numismática de la Argentina y el Instituto Histórico y Geográfico de Uruguay en torno a la fundación del Instituto, sus fines y las distintas actividades. El fortalecimiento de esas relaciones se puso de manifiesto en una correspondencia que iría in crescendo referida a intercambios bibliográficos, de documentos y de fuentes de información; hacia mediados de la década de 1940 se iniciaron estudios para la aplicación de convenios sobre reciprocidad de credenciales académicas. Consta, asimismo, que a las primeras sesiones del Instituto asistían personalidades públicas y 
funcionarios que, eventualmente, contribuyeron a allanar esos intercambios, como por ejemplo el representante diplomático de la República Argentina en Paraguay, Juan Carlos Valenzuela, y el agregado comercial de dicha legación, José Rodríguez Alcalá. Un primer corolario en ese sentido fue la designación de Adolfo Aponte como miembro correspondientes de la Junta de Historia y Numismática de la Argentina.

La segunda línea de acción se dirigió a promover el aumento de miembros de número y correspondientes. Entre 1937 y 1955 se designaron 46 numerarios y fueron nombrados en el exterior 24 correspondientes, entre los que figuraban Emilio Ravignani, Ricardo Levene y Ramón de Castro Estévez en Argentina, Silvio Zavala en México, José Carlos de Macedo Soares y Virgilio Correa Filho — presidente y secretario del Instituto Histórico y Geográfico- y Walter Z. de Azevedo en Brasil, Alcides Arguedas en Bolivia, Raimundo Rivas y José Arango en Colombia, Juan Manuel Carbonell en Cuba, Manuel Caviezes Serrano y Carlos Grez Pérez en Chile, Ernesto J. Castillero en Panamá y Pedro Dulanto en Perú.

Si se atiende a la nómina de los que se incorporaron como numerarios en esa época puede visualizarse a un conjunto de hombres que coincidían en espacios de actuación política y cultural. Julio César Chaves, por ejemplo, fue quien organizó durante la guerra del Chaco el Departamento de Prensa y Propaganda, y en 1938 accedió a una banca en la cámara de Diputados; Cecilio Báez y Efraím Cardozo fueron representantes del Paraguay en la firma del Tratado de Paz y Amistad con Bolivia, celebrado en Buenos Aires el 21 de junio de 1938; José Félix Estigarribia, quien también formó parte de esa representación paraguaya, se incorporó al Instituto en 1939, un año antes de asumir la presidencia del país; Justo Pastor Benítez y Pablo Max Insfrán fueron los redactores de la controvertida constitución de 1940, sancionada bajo la administración de Estigarribia. Todos estos militaban activamente en el partido Liberal y se dedicaban a una intensa actividad periodística.

Dentro de ese conjunto, los miembros fundadores R. Antonio Ramos e Hipólito Sánchez Quell junto a dos de los hombres que se incorporaron en 1938, Julio César Chaves y Efraím Cardozo, compondrían un grupo de fuerte influencia en la vida institucional. Esto se debió en primer término a su cuantiosa producción histórica en relación a la de los otros integrantes del Instituto, puesta de manifiesto ya en la época de la fundación. Chaves publicó en 1937 Historia de las relaciones entre Buenos Aires y el Paraguay y en 1941 El Supremo Dictador; también en ese último año 
INSTITUCIONALIZAR LA ESCRITURA DEL PASADO

NÓMINA DE MIEMBROS DE NÚMERO INCORPORADOS AL INSTITUTO

PARAGUAYO DE INVESTIGACIONES HISTÓRICAS ENTRE 1937 Y 1956

\begin{tabular}{|c|c|c|}
\hline Año & Incorporaciones & Total \\
\hline 1937 & $\begin{array}{l}\text { Adolfo Aponte } \\
\text { Ramón Indalecio Cardozo } \\
\text { Hipólito Sánchez Quell } \\
\text { R. Antonio Ramos } \\
\text { Ramón Lara Castro } \\
\text { Eduardo Amarilla Fretes } \\
\text { Carlos R. Centurión } \\
\text { Viriato Díaz Pérez } \\
\text { Arturo Brugada } \\
\text { José Gabriel Ruiz }\end{array}$ & 10 \\
\hline 1938 & $\begin{array}{l}\text { Julio César Chaves } \\
\text { Andrés Barbero } \\
\text { Cecilio Báez } \\
\text { José Félix Estigarribia } \\
\text { Juan Manuel Sosa Escalada } \\
\text { Enrique Bordenave } \\
\text { Efraím Cardozo } \\
\text { Modesto Guggiari } \\
\text { Benjamín Velilla } \\
\text { Tomás Osuna } \\
\text { César Vasconcellos }\end{array}$ & 11 \\
\hline 1939 & $\begin{array}{l}\text { Pablo Max Insfrán } \\
\text { Justo Pastor Benítez } \\
\text { Arsenio López Decoud } \\
\text { Juan B. Otaño } \\
\text { Juan Francisco Pérez Acosta }\end{array}$ & 5 \\
\hline 1940 & $\begin{array}{l}\text { Pablo Alborno } \\
\text { Narciso Colman } \\
\text { J. Natalicio González } \\
\text { Justo Prieto } \\
\text { Benigno García Riquelme } \\
\text { Casaccia Bibolini, Benigno } \\
\text { Guillermo Tell Bertoni } \\
\text { José Doroteo Bareiro }\end{array}$ & 8 \\
\hline
\end{tabular}

Fuente: elaboración propia sobre la base de archivo de la Academia Paraguaya de la Historia. 
NÓMINA DE MIEMBROS DE NÚMERO INCORPORADOS AL INSTITUTO PARAGUAYO DE INVESTIGACIONES HISTÓRICAS ENTRE 1937 Y 1956 (cont.)

\begin{tabular}{|c|l|c|}
\hline Año & Incorporaciones & Total \\
\hline 1941 & $\begin{array}{l}\text { Juan Ramón Chaves } \\
\text { Emilio Saguier Aceval } \\
\text { Raimundo Rolón } \\
\text { Alcides Codas Papaluca }\end{array}$ & 4 \\
\hline 1944 & $\begin{array}{l}\text { Juan Emiliano O'Leary } \\
\text { Silvano Mosqueira } \\
\text { Marciano Castelví } \\
\text { Marco Antonio Laconich }\end{array}$ & \\
\hline 1947 & $\begin{array}{l}\text { Juan Bautista Gill Aguinaga } \\
\text { Mariano Luis Lara Castro } \\
\text { Carlos A. Pastore }\end{array}$ & 3 \\
\hline 1950 & Juan Bautista Rivarola & 1 \\
\hline & & Total: 46 \\
\hline
\end{tabular}

apareció La fundación de la ciudad de Asunción, de Efraím Cardozo; en 1944 comenzó a circular La política del Brasil bajo la dictadura del doctor Francia, de R. Antonio Ramos, obra a la que seguirían La política del Brasil en Paraguay y Juan Andrés Gelly. La segunda circunstancia fue el rol activo que el grupo iría desenvolviendo en el espacio universitario. En efecto, el 16 de febrero de 1948 se fundó la Facultad de Filosofía de la Universidad Nacional de Asunción en la que quedó habilitada la sección Historia y comenzó a dictarse la carrera de grado y el doctorado. ${ }^{17}$ Efraím Cardozo obtendría la cátedra de Historia Colonial, R. Antonio Ramos la de Historia Americana y Julio César Chaves la de Historia Diplomática del Paraguay.

Una tercera circunstancia que permite sostener el fuerte influjo de esos hombres es la trama institucional que concertaron con el Archivo Nacional de Asunción, del que R. Antonio Ramos fue director. ${ }^{18}$

17 Caballero Campos, 2009, v. II, 15-36; Monte de López Moreira, 2012, 12-65.

18 Viriato Díaz Pérez y Juan E. O’Leary, miembros del IPIH, también ocuparon la dirección del Archivo Nacional. Existen constancias, aunque no se ha producido aún una indagación específica, de que el archivo era un espacio destacado de sociabilidad entre los letrados asuncenos durante la primera mitad del siglo XX. 


\section{La segunda etapa}

Desde el final de la guerra del Chaco en 1935 hasta el año 1954 Paraguay fue gobernado por diez presidentes. En 1947 se desarrolló una sangrienta lucha civil que catapultó al poder al partido Colorado aunque la ruptura institucional se prolongaría hasta que el general Alfredo Stroessner se convirtió en presidente después de un golpe militar el 4 de mayo de 1954. Como resultado de esos enfrentamientos no pocos de los integrantes del IPIH, afiliados en muchos casos, como se ha referido, al partido Liberal, tomaron el camino del exilio: Carlos Pastore lo hizo en Uruguay; Pablo Max Insfrán en Estados Unidos; Efraím Cardozo, Julio César Chaves y Justo Pastor Benítez en Buenos Aires. Estas circunstancias explican que el IPIH no pudiera desarrollar las sesiones con regularidad y que no se produjera ningún ingreso como miembro de número o correspondiente entre 1950 y 1956.

En 1952 regresó a Paraguay Julio César Chaves y dos años después lo hizo Efraím Cardozo. Así las cosas, el 11 de agosto de 1956 Chaves y otros integrantes del IPIH convocaron a una asamblea extraordinaria en cuyo transcurso se adoptaron importantes resoluciones. En primer término fue electa una nueva comisión directiva presidida por Chaves e integrada por: Vicepresidente 1..$^{\circ}$ Juan Bautista Gill Aguinaga; Vicepresidente 2. ${ }^{\circ}$ Marco Antonio Laconich; Secretarios Benigno Riquelme García y Dr. Luis Mariano Castro; Tesorero Juan Bautista Rivarola; Académicos Consejeros Benjamín Velilla, Raimundo Rolón, Efraím Cardozo, Hipólito Sánchez Quell, Carlos R. Centurión y R. Antonio Ramos; y Consejeros suplentes Carlos Alberto Pusineri y Carlos Guggiari Alfaro.

Luego se votó positivamente la reforma del artículo $4 .^{\circ}$ del estatuto general del Instituto por el que se elevó a 45 la cantidad de numerarios, en lugar de los 40 que se establecieron originalmente. Con esta modificación, en el transcurso de ese año se designaron 16 miembros de número entre los cuales estuvo la primera mujer: la escritora María Concepción Leyes de Chaves (1891-1985), quien tenía en prensa una biografía novelada sobre Elisa Lynch, y sería por bastante tiempo la única presencia femenina en el Instituto. ${ }^{19}$

19 Nuevos ingresos femeninos se producirían recién a partir de los años setenta: Idalia Flores de Zarza (1977), Olinda Massare de Kostianovsky, Julia Velilla Laconich y Beatriz Rodríguez Alcalá (1978). 
Con el fin de intensificar los lazos con Academias e historiadores del exterior, la nueva comisión directiva adoptó dos decisiones. La primera consistió en designar un número significativo de miembros correspondientes. En España a Emilio García Gómez, Manuel Ballesteros, Guillermo Céspedes, Octavio Gill Munilla, Manuel García Blanco, Francisco Sevillano Colombo y Jaime Delgado; en Argentina a Raúl Molina, Ricardo Caillet Bois y Ricardo Zorraquín Becú; en Bolivia a Humberto Vázquez Machicado, Augusto Guzmán, Hernando Fernández Sanabria, Armando Alba y Gunnar L. Mendoza; en Brasil a Guy de Hollanda y Rosendo Sampaio García; en Chile a Ricardo Donoso; en Colombia a Manuel José Forero; en Guatemala a Adrián Racinos; en Nicaragua a Adolfo Calero Orozco; en Honduras a Eufemiano Claro; en Panamá a Ricardo J. Alfaro; en Puerto Rico a Washington Lorens; en Perú a Raúl Porras Barrenechea; en Venezuela e Jesús A. Cova y en Estados Unidos a John P. Harrison.

Se decidió, asimismo, que el presidente Chaves realizara un viaje a Europa con el propósito de afianzar contactos internacionales. De ese periplo han quedado escasas constancias, entre las que figuran noticias de la entrevista que mantuviera en París con Pierre Chaunu - director del Instituto de Altos Estudios Latinoamericanos-, de las conversaciones en Italia con las autoridades de la Biblioteca Ambrosiana, y en España con miembros de la Real Academia de la Historia.

En las Actas de sesiones del año 1956 puede leerse que el presidente de la corporación fijó el rol que, según entendía, en adelante le cabía a la corporación: «ojalá que en esta casa se forme y de esta casa salga la futura gran generación de historiadores nacionales. Ningún servicio más alto podíamos rendir al país». Al mismo tiempo señalaba las rémoras que, en su opinión, ensombrecían la práctica de la historia en Paraguay:

\footnotetext{
Todavía se pretende unir a la historia, que es la mayor riqueza de los paraguayos, a los ajetreos de la politiquería, a menguados intereses, a bastardas ambiciones [...] Todavía ese archivo nuestro [Archivo Nacional de Asunción] vive allí como un huérfano desamparado, sin amparo y sin protección, corriendo graves peligros el invalorable fondo documental que encierra. Todavía las riquezas artísticas dejan nuestros museos y templos para ir a enriquecer colecciones particulares y pinacotecas oficiales del exterior.
}

Pues bien, a la notoriedad que en esos años habían adquirido algunos de sus miembros debido a su profusa producción histórica y la dedicación más exclusiva al estudio de la historia que suponía el desarrollo de la 
carrera universitaria, se sumaba el eco que iban encontrando las actividades culturales propuestas por el Instituto, puesto de manifiesto en la alta concurrencia de público.

Todas esas circunstancias confluían para que en el futuro inmediato el Instituto tuviera, siguiendo las expresiones de Chaves, «una gran misión que cumplir, una tarea vigilante que realizar». Al hilo de esa frase programática debe situarse la aparición en 1956 del primer volumen de Historia Paraguaya.

NÓMINA DE MIEMBROS DE NÚMERO INCORPORADOS AL INSTITUTO PARAGUAYO DE INVESTIGACIONES HISTÓRICAS ENTRE 1956 Y 1966

\begin{tabular}{|c|l|c|}
\hline Año & Incorporaciones & Total \\
\hline 1956 & $\begin{array}{l}\text { Juan Max Boettner } \\
\text { Félix Paiva } \\
\text { Marcelino Noutz } \\
\text { Manuel Riquelme } \\
\text { Emiliano Gómez Ríos } \\
\text { Jesús Blanco Sánchez } \\
\text { José Concepción Ortiz } \\
\text { Cipriano Codas } \\
\text { Osvaldo Chaves } \\
\text { Roque Gaona } \\
\text { Gustavo González } \\
\text { Carlos A. Guggiari Alfaro } \\
\text { Benjamín Vargas Peña } \\
\text { Carlos Alberto Pusineri Scala } \\
\text { César Alonso de las Heras } \\
\text { María Concepción Leyes de } \\
\text { Chaves }\end{array}$ \\
\hline 1958 & Manuel Peña Villamil & \\
\hline 1959 & $\begin{array}{l}\text { Alberto Nogués } \\
\text { Pastor Urbieta Rojas } \\
\text { Rafael Eladio Velázquez }\end{array}$ \\
\hline & \\
\hline
\end{tabular}




\section{Historia Paraguaya}

Es conocido que en los primeros años de la posguerra del Chaco se produjo una retracción en materia de publicaciones en el Paraguay. Para las principales editoriales que habían funcionado hasta esa fecha como La Colmena, La Mundial y La Librería de Puigbonet, la coyuntura política delimitada por la ruptura institucional, el estado de sitio y la intervención de la prensa afectaron su rentabilidad. No pocos escritores paraguayos buscaron talleres gráficos extranjeros, sobre todo argentinos, que les ofrecían condiciones económicas más ventajosas. En Buenos Aires comenzaron a funcionar editoriales paraguayas o dedicadas exclusivamente a lo paraguayo; alcanzaron auge las denominadas Ayacucho, fundada por Julio César Chaves, Guarania, establecida por Juan Natalicio González, y Tupá, dirigida por Anselmo Jover Peralta. Con el sello de Ayacucho se editaron en la capital argentina los reconocidos estudios de los académicos Justo Pastor Prieto, La vida indómita de Augusto Comte (1944); de Justo Pastor Benítez, El Solar guaraní (1947); y de Carlos Centurión, Historia de las letras paraguayas (1951), entre otros. Cuando regresó de su exilio Julio César Chaves le propuso a un librero italiano instalado en Asunción lanzar las «ediciones Nizza» y él mismo inició la publicación de la Biblioteca Histórica Paraguaya de Cultura Popular.

En ese contexto, la aparición en 1956 del primer volumen del Anuario del IPIH, titulado Historia Paraguaya, merece ser subrayada. Era la primera publicación periódica especializada en historia que aparecía en mucho tiempo y, no obstante algún retraso, saldría con puntualidad encomiable.

Sus secciones fijas las constituían artículos de los miembros del Instituto, la divulgación de documentos históricos, de poesías y de reseñas bibliográficas. Por lo demás, en algunos volúmenes se incluyó una sección dedicada a la crónica académica, en tanto que los discursos de ingreso comenzaron a ser publicados a partir del año 1966, cuando se lo dispuso como condición para la incorporación efectiva de los nuevos miembros. Fue la única publicación de estas características que circuló en el país hasta que en el año 1964 comenzó a editarse la Revista Paraguaya de Sociología. Y, según la reseña sobre la producción histórica paraguaya que ofreciera John Hoyt Williams a comienzos de la década del setenta, eran las dos únicas revistas «dignas de consulta». ${ }^{20}$

20 Williams, 1973. 
Entre 1956 y 1965 vieron la luz diez volúmenes de Historia Paraguaya. Un recuento de los artículos publicados da cuenta de que las temáticas referidas al período hispánico, al proceso de la independencia y a la primera mitad del siglo XIX fueron las que más atrajeron a los integrantes del IPIH y las que constituyeron el mayor aporte a la historiografía paraguaya. La historia política, diplomática e institucional eran, por su parte, los campos en los que puede situarse la mayoría de los estudios históricos allí publicados.

HISTORIA PARAGUAYA 1956-1965

\begin{tabular}{|l|c|}
\hline Temas & Artículos \\
\hline Población aborigen & 2 \\
\hline Período hispánico & 14 \\
\hline Revolución de la independencia & 10 \\
\hline Gobiernos de la primera mitad del siglo XIX (historia política) & 7 \\
\hline Guerra contra la Triple Alianza & 1 \\
\hline $\begin{array}{l}\text { Temas de historia cultural (educación, folklore, instituciones } \\
\text { culturales) }\end{array}$ & 7 \\
\hline
\end{tabular}

Luego de una década de ininterrumpida publicación de Historia Paraguaya y como consecuencia del desarrollo alcanzado por el Instituto después de más de veinte años de labor cultural y del reconocimiento que había adquirido la producción histórica de sus miembros, en la Asamblea del 28 de octubre de 1965, por el voto unánime de los miembros de número y correspondientes, se aprobó una resolución por la que el IPIH se transformó en Academia Paraguaya de la Historia.

Un nuevo estatuto, establecido en 1966, desplegaba como fines:

Propulsar los estudios historiográficos en el país, defender y enaltecer el acervo histórico de la nación y propiciar la publicación de los clásicos de la historiografía nacional; auspiciar congresos, conferencias, coloquios, concursos, etc. sobre la materia; mantener vinculaciones con instituciones del país y del exterior; publicar revistas especializadas y auspiciar la edición de las obras de los académicos y colaborar con las autoridades nacionales en la elaboración de los planes de estudio de la Educación. 
La Academia quedaba constituida, a diferencia de otros casos en América, como una institución civil no oficial. ${ }^{21}$ Estaría compuesta por 35 académicos de número y por un número indeterminado de miembros correspondientes tanto en el país como en el extranjero. En adelante, para ser nombrado académico de número o correspondiente se requería «haberse destacado en la especialidad con investigación comprobada y ser propuesto por escrito por un mínimo de cinco académicos de número». Y para ser incorporado debería pronunciar en solemne sesión pública una conferencia que constituyese «un trabajo original y de investigación comprobada».

Las autoridades serían elegidas cada dos años en asamblea general ordinaria y estarían compuestas por presidente, vicepresidente, secretario, tesorero, bibliotecario-archivero y síndico.

Podría sostenerse que la nueva figura institucional reflejaba algo más trascendente que una modificación de su estructura. La transformación era el resultado, en buena parte, de la acción de una pléyade de historiadores paraguayos cuyos estudios históricos comenzaron a hacerse visibles en los años veinte, cuyo prestigio fue acrecentándose mediante la introducción de un tratamiento cuidadoso de las fuentes históricas en sus investigaciones y de una dedicación más exclusiva a la historia a través de las cátedras universitarias. Son algunos de ellos los que a su vez retendrán los cargos directivos de la Academia: Julio César Chaves fue presidente entre 1956 y 1972 y de 1984 a 1986, R. Antonio Ramos entre 1976 y 1978 y luego entre 1982 y 1984 e Hipólito Sánchez Quell, entre 1978 a 1980.

El joven historiador Rafael Eladio Velázquez (1926-1994), cercano a Cardozo, se incorporó al Instituto en 1959, a su regreso de Sevilla, donde había cursado estudios de Historia del Derecho luego de obtener el doctorado en Historia en la Universidad Nacional de Asunción. ${ }^{22}$ Velázquez dejó constancia de las circunstancias en las que emergió la APH y de sus principales protagonistas. En efecto, se conserva en su archivo personal un conjunto de fichas manuscritas con el título de «Historiografía Paraguaya», en las que resume sus impresiones sobre el conjunto de autores que, según entendía, desde mediados del siglo XX habían «sentado escuela» por su rigurosa metodología. El criterio para reunirlos era que cada uno de ellos constituía un «hito» en la construcción de una «interpretación honesta y

21 Es decir que no fue establecida por un decreto presidencial como habían sido los casos de la Academia Nacional de la Historia de Venezuela (1888), la Academia Nacional de la Historia de Perú (1905) y la Academia Colombiana de la Historia (1902).

22 Velázquez, 1958. 
seria de la historia paraguaya» que se sustentaba en un avance del rigor heurístico. Figuran en ese grupo los académicos Pablo Max Insfrán, Carlos R. Centurión, R. Antonio Ramos y Efraím Cardozo. Entre ellos, dedicó especiales referencias a este último concentrándose en dos de sus iniciativas: la «monumental Historiografía Paraguaya», que publicara en México en 1959, y el plan para una historia general del Paraguay. ${ }^{23}$ Velázquez destacaba en el manuscrito tanto la prolífica labor historiográfica de Cardozo como su talante como historiador, puesto que — sostenía - «no vivió enclaustrado en su gabinete de trabajo sino que participó activamente de nuestras luchas cívicas y prodigó su salud en la cátedra y el periodismo».

En cuanto al proyecto de una historia general del Paraguay, Velázquez sostenía que se disponía de pruebas que demostraban que Cardozo pergeñó el proyecto en los años 60, como una primera síntesis histórica del país. El propósito quedaría trunco con su muerte en 1973, no obstante haber dejado finalizada la redacción de ocho capítulos, correspondientes a los siglos XVI y XVII, en los que analizaba el territorio, los primitivos habitantes, el descubrimiento, la creación, la conquista, las fundaciones, el gobierno de Hernandarias, las consecuencias de la división territorial de 1617 y la destrucción del Guairá. El manuscrito pone en evidencia que se trató de un plan con el que Cardozo se proponía llegar al mayor número posible de lectores, motivo por el cual limitó sustancialmente el uso de notas y de referencias de fuentes documentales. A su vez, el contenido aparece como corolario de una producción histórica sostenida en el tiempo que Cardozo principiara en 1949 - como ha sido, en parte, ya apuntado - con la publicación de El Paraguay Independiente para luego añadir en los años siguientes las obras El Paraguay Colonial (1955), Historiografía Paraguaya (1959), Historia Cultural del Paraguay (1964) y Breve Historia del Paraguay (1965). ${ }^{24}$ La suma de los intereses cultivados puestos de manifiesto en esos estudios, además de su esencial continuidad, permitirían sostener, con toda probabilidad, que Cardozo fue el historiador más «total» - en el sentido de la cantidad de intereses y territorios históricos que fueron objeto de sus indagaciones - de la Academia Paraguaya de la Historia al momento de su fundación, sin dejar de advertir, claro está, la necesidad de un análisis más exhaustivo en torno a este supuesto.

23 República del Paraguay, Archivo de la Academia Paraguaya de la Historia, Fondo Rafael Eladio Velázquez (APH-FREV), fichas manuscritas, sin fecha.

24 Esos materiales serían finalmente publicados en el año 1989 bajo el título de El Paraguay de la Conquista. 


\section{Conclusiones}

Hasta aquí el breve recuento de las circunstancias que rodearon a la fundación de la Academia Paraguaya de la Historia y la aproximación a quienes la integraron entre 1937 y 1965. De intento nos hemos movido en un nivel descriptivo por lo que este estudio no está agotado. Se trata únicamente de un punto de partida para una investigación que, en un futuro cercano, cubra los jalones posteriores a ese último año.

No obstante, el trayecto expuesto en este artículo hace posible enumerar algunas conclusiones. En primer término puede concluirse que la Academia Paraguaya de la Historia fue promovida, al igual que la mayoría de las academias en América, por un grupo de letrados deseosos de lograr progresos en los estudios históricos nacionales; surgió como un cenáculo que contribuyó a crear un nuevo tipo de espacio público-privado en Paraguay, diferente al estatal y al estrictamente privado o íntimo, en el que se reunía un grupo de personas para conversar y hacer uso de su capacidad crítica racional.

Sus fundadores y los miembros que se incorporaron hasta el año 1965 no fueron, con la excepción de Rafael Eladio Velázquez, historiadores profesionales, es decir, formados específicamente en la disciplina en el espacio universitario, pero dedicaron a la escritura de la historia buena parte de sus esfuerzos intelectuales.

Entre los componentes del grupo un hombre cobra importancia al facilitar, con toda probabilidad, la articulación y cohesión: Julio César Chaves. No solo por su prolífica producción sino también porque fue quien impulsó la edición de Historia Paraguaya y profundizó el relacionamiento regional e internacional de la institución.

La historia política, diplomática e institucional fueron los territorios que más atrajeron a los académicos, concentrándose en temáticas referidas sobre todo al período hispánico y a la primera mitad del siglo XIX. Sus intereses no se vieron modificados desde su ingreso a la Academia, más bien se nota una continuidad y profundización, si para ello se tiene en cuenta las obras que publicaron entre 1937 y 1965.

Una mayor rigurosidad en el tratamiento de las fuentes por parte de los historiadores de la Academia destaca como un avance cualitativo para la práctica de la historia en el Paraguay desde mediados del siglo XX, aunque hay que decir al mismo tiempo que, según las pruebas disponibles hasta ahora, tanto los textos publicados en Historia Paraguaya como los 
estudios monográficos individuales no se habrían hecho eco de las transformaciones que fueron acompañando la práctica de la historia en esos años en Europa y en algunos países de América.

Contando con estos avances aparece asimismo como necesidad fundamental establecer de qué modo el contexto político caracterizado por el régimen dictatorial de Alfredo Stroessner condicionó el proceso de institucionalización de la historia en Paraguay a partir de los años sesenta. Como ha sido mostrado en investigaciones recientes referidas, por ejemplo, al caso de la institucionalización de la sociología, el régimen desmovilizó a la sociedad civil y puso especial énfasis en la reducción del espacio público y la ampliación del sector privado. Teniendo esto en cuenta podría cavilarse que la Academia Paraguaya de la Historia se constituiría en un factor de institucionalización destacado.

Recibido el 19 de mayo de 2014 Aceptado el 20 de noviembre de 2015

\section{Bibliografía}

Amaral, Raúl: El Novecentismo paraguayo: hombres e ideas de una generación fundamental del Paraguay, Asunción, Servilibro, 2006.

Betancourt Mendieta, Alexander: Historia y Nación, Medellín, La carreta histórica, 2007.

Betancourt Mendieta, Alexander y Ramírez Bacca, Renzo (coords.): Miradas de contraste. Estudios comparados sobre Colombia y México, México, Porrúa, 2009.

Bouvier, Virginia: El ocaso de un sistema. Encrucijada en Paraguay, Asunción, Intercontinental, 2012.

Brezzo, Liliana M.: «Paraguay: la historia y los historiadores», en Telesca, Ignacio (coord.), Historia del Paraguay, Asunción, Taurus, 2010a, 13-32.

Brezzo, Liliana M.: «Reparar la Nación. Discursos históricos y responsabilidades nacionalistas en Paraguay», Historia Mexicana, LX, México, 2010b, 197242.

Brezzo, Liliana M.: «Representaciones del Paraguay en el Centenario», en Meliá, Bartomeu (coord.), Otras historias de la Independencia, Asunción, Taurus, 2011, 39-63.

Caballero Campos, Herib (coord.): Universidad Nacional de Asunción. 120 años de Historia, Asunción, Ed. Pri, 2009, 3 vols.

Cardozo, Efraím: Historiografía Paraguaya, México, IPGH, 1959. 
Catálogo detallado en secciones de la Biblioteca de José Segundo Decoud. Estadista del Paraguay, Buenos Aires, Spinelli, 1912.

Centurión, Carlos: Historia de la cultura paraguaya, Asunción, Biblioteca Ortiz Guerrero, 1961, 2 tomos.

Devoto, Fernando y Pagano, Nora: Historia de la historiografía argentina, Buenos Aires, Sudamericana, 2009.

Durán Estragó, Margarita (comp.): La Aurora. Enciclopedia mensual y popular de artes, ciencias y literatura, Asunción, FONDEC, 2006.

Gómez Lez, Osvaldo y Zarza, Miguel (comps.): Pensadores (as) del 900, Asunción, CIF-Secretaría Nacional de Cultura, 2013.

Hampe Martínez, Teodoro: «Trayectoria y balance en la historiografía peruana: 90 años de la Academia Nacional de la Historia (1905-1995)», Anuario de Estudios Americanos, LV, 2, Sevilla, 1998, 703-725

Instituto Paraguayo de Investigaciones Históricas: Historia Paraguaya, Asunción, IPIH, 1956-65, 10 vols.

Massare de Kostianovsky, Olinda: «Aporte documental para una historia de la Academia Paraguaya de la Historia. Defensor del patrimonio histórico del país», Historia Paraguaya, XLVII, Asunción, 2007, 349-383.

Micheletti, María Gabriela: «Blasones intelectuales, lecturas regionales, derivas nacionales. Aportes santafesinos a la historiografía académica argentina (1893-1938)», Anuario de Estudios Americanos, 70, 1, Sevilla, 2013, 221248.

Monte de López Moreira, Mary (coord.): Evolución histórica de la Facultad de Filosofía de la Universidad Nacional de Asunción, Asunción, UNA, 2012.

Nava Rodríguez, María Teresa: «La Real Academia de la Historia como modelo de unión formal entre el Estado y la Cultura (1735-1792)», Cuadernos de Historia Moderna y Contemporánea, 8, Madrid, 1987, 127-155.

Rodríguez, José Carlos: El Paraguay bajo el nacionalismo, Asunción, El Lector, 2010.

Scavone Yegros, Ricardo: «Guerra internacional y confrontaciones políticas (1920-1954)», en Telesca, Ignacio (coord.), Historia del Paraguay, Asunción, Taurus, 2010a, 223-265.

Scavone Yegros, Ricardo: Polémicas en torno al gobierno de Carlos Antonio López en la prensa de Buenos Aires 1857-1858, Asunción, Tiempo de Historia, 2010b.

Schmitt, Peter: Paraguay y Europa 1811-1870, Asunción, La Pluma, 1990.

Vázquez, Josefina Zoraida: «Cincuenta y tres años de las Memorias de la Academia Mexicana de la Historia», Historia Mexicana, L, 4, México, 2001, 709-718.

Velázquez, Rafael Eladio: «Paraguay», Anuario de Estudios Americanos, XV, Sevilla, 1958, 675-686. 


\section{INSTITUCIONALIZAR LA ESCRITURA DEL PASADO}

Vidaurreta, Alicia: «El Paraguay a través de viajeros, 1843-1917», Estudios Paraguayos, 11, 1, Asunción, 1972, 51-89.

Whigham, Thomas L. y Scavone Yegros, Ricardo: José Falcón. Escritos Históricos, Asunción, Servilibro, 2006.

Williams, John Hoyt: «Del calor al frío. Una visión personal de la historiografía paraguaya», Estudios Paraguayos, 1, Asunción, 1973, 139-163. 